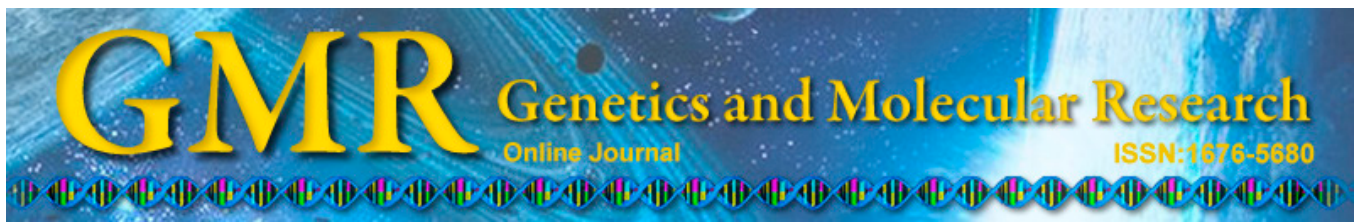

\title{
Association between adiponectin receptor 2 gene polymorphisms and cerebral infarction
}

\author{
B. Yuan ${ }^{1,2}$ and J.F. Teng ${ }^{1}$ \\ ${ }^{1}$ Department of Neurology, First Affiliated Hospital of Zhengzhou University, \\ Zhengzhou, China \\ ${ }^{2}$ No. 2 Department of Neurology, \\ First Affiliated Hospital of Xinxiang Medical University, Xinxiang, China \\ Corresponding author: J.F. Teng \\ E-mail: junfangt@yeah.net
}

Genet. Mol. Res. 13 (3): 7808-7814 (2014)

Received May 6, 2014

Accepted September 12, 2014

Published September 26, 2014

DOI http://dx.doi.org/10.4238/2014.September.26.19

\begin{abstract}
We examined the association between the adiponectin receptor 2 gene and the risk of ischemic stroke. Polymerase chain reaction-restriction fragment length polymorphism was used to detect rs12342 genotypes of the adiponectin receptor 2 gene in 300 ischemic stroke patients and 320 age- and gender-matched healthy controls. In the patient group, the AA, GA, and GG genotype frequencies were $39.3,42.7$, and $18.0 \%$, respectively. The $\mathrm{A}$ and $\mathrm{G}$ allele frequencies were 0.607 and 0.393 , respectively. In the control group, the AA, GA, and GG genotype frequencies were $29.0,51.7$, and $19.3 \%$, respectively. The $\mathrm{A}$ and $\mathrm{G}$ allele frequencies were 0.548 and 0.452 , respectively. The AA genotype and A allele frequencies in the patient group were significantly higher than those in the control group (both $\mathrm{P}<0.01$ ). The risk of ischemic stroke in AA genotype carriers was 1.786-fold greater than that in GG genotype carriers (odds ratio $=1.786,95 \%$ confidence interval: 1.432-2.775; $\mathrm{P}=0.013$ ). After adjusting for various confounding factors, the difference remained significant (odds ratio $=1.874,95 \%$ confidence interval: $1.221-2.765 ; \mathrm{P}=0.012)$. The AA genotype and A allele of rs12342 in the adiponectin receptor 2
\end{abstract}


gene may increase the risk of ischemic stroke, particularly the risk of atherosclerosis cerebral infarction.

Key words: Adiponectin receptor 2 gene; Ischemic stroke; Single nucleotide polymorphisms

\section{INTRODUCTION}

Atherosclerosis is an important pathological mechanism in the incidence of ischemic stroke. Adiponectin is a fat factor with protective effects in the body, such as anti-atherosclerotic and anti-inflammatory properties. It also increases insulin sensitivity and maintains energy balance, glucose metabolism, and lipid metabolism, which are closely related with the incidence of ischemic stroke (Hewitt et al., 2012). Adiponectin can regulate glucose and lipid metabolism through its specific receptor to exert its anti-inflammatory and anti-atherosclerotic effects (Baranowska et al., 2011; Hewitt et al., 2012). In rhesus monkey animal models, adiponectin levels decreased, increasing insulin resistance and deterioration of type 2 diabetes (Wahab et al., 2010). A previous study indicated that hypoadiponectinemia is involved in insulin resistance and type 2 diabetes in animal models (Hotta et al., 2001). Kubota et al. (2006) applied gene knockout technology and found that compared with wild-type mice, adiponectin knockout heterozygous (adipo+/-) mice showed mild insulin resistance. Homozygous (adipo-/-) mice showed moderate insulin resistance and impaired glucose tolerance. These results indicate that adiponectin plays a very important role in the maintenance of insulin sensitivity and normal glucose metabolism. In vitro studies have shown that adiponectin can inhibit the expression of adhesion molecules of tumor necrosis factor $\alpha$ (Kabara et al., 2014) and the monocytes from adhering to endothelial cells (Yang et al., 2013), reduce the expression of scavenger receptor type A and lipid accumulation on the surface of macrophages, and inhibit macrophage transformation into foam cells (Tian et al., 2012). In addition, adiponectin can inhibit damaged vascular intimal hyperplasia, smooth muscle cell proliferation, and migration to reduce plaque size (Uemura et al., 2013). Animal experiments further confirmed the anti-inflammatory and anti-atherosclerotic effects of adiponectin (Du et al., 2013). Because adiponectin has a variety of biological effects through adiponectin receptors, the adiponectin receptor gene (ADIPOR) may be a susceptibility gene for ischemic stroke. However, the relationship between ADIPOR and the risk of ischemic stroke remains unclear. In this study, we examined the association between rs 12342 in the ADIPOR 2 gene and the risk of ischemic stroke in a Chinese population.

\section{MATERIAL AND METHODS}

\section{Subjects}

We enrolled 300 patients with ischemic stroke who were treated in the Department of Neurology, First Affiliated Hospital of Xinxiang Medical College from September 2008 to May 2013 in this study. Among these 300 patients, there were 191 males and 109 females whose ages ranged from 35 to $85(65.1 \pm 11.3)$ years. The diagnosis of ischemic stroke in all patients was confirmed by brain magnetic resonance imaging and/or head computed tomography. Patients with cerebral embolism, arterial inflammation, malignancy, trauma, drugs, blood 
disease, cerebral infarction caused by vascular malformation or aneurysm, liver and kidney disease, or thyroid disease were excluded from the study. According to trial of ORG 10172 in acute stroke treatment classification criteria, these 300 patients were divided into an atherosclerotic cerebral infarction group and a lacunar infarction group.

During the same period, 320 healthy individuals in the medical center of the same hospital were selected as the control group. All control subjects were unrelated Han people who were age- and gender-matched with the patient group. There were 201 male and 119 female subjects ranging in age from 35 to $87(65.5 \pm 11.1)$ years. The subjects with cerebrovascular disease, neurological diseases, kidney disease, blood disorders, cancer, peripheral vascular disease, and autoimmune diseases were excluded from the control group. These control subjects had no history of cerebrovascular disease and no signs of cerebrovascular disease based on computed tomography or magnetic resonance imaging scanning. Clinical characteristics, including age, gender, height, weight, blood pressure, lipid profiles, fasting glucose, medical history, drug history, smoking history, and alcohol history, were collected. All study subjects signed informed consent forms before participating in the study.

\section{Blood collection and DNA extraction}

First, $2 \mathrm{~mL}$ fasting venous blood was taken from the antecubital vein and placed in ethylenediaminetetraacetic acid-containing tubes. A genomic DNA extraction kit (Promega Corporation; Madison, WI, USA) was used for DNA extraction from blood samples according to the manufacturer protocol.

\section{Primer design}

Primers were designed using the Primer 5.0 software (Premier Biosoft; Palo Alto, CA, USA). The primers had the following sequences: upstream primer: 5'-CAAGGGCAAGGGAG GAAA-3', downstream primer: 5'-CAGGGAGTGAGGTACAAGACGA-3'.

\section{Genotyping}

The polymerase chain reaction (PCR)-restriction fragment length polymorphism method was used to perform the genotyping. PCR amplification was conducted in a volume of $25 \mu \mathrm{L}$, including: $1 \mu \mathrm{L} 200 \mathrm{ng} / \mu \mathrm{L}$ template DNA, $0.5 \mu \mathrm{L}$ of each $20 \mu \mathrm{M}$ up- and downstream primer, $2 \mu \mathrm{L}$ dNTP mixture, $0.125 \mu \mathrm{L}$ Taq polymerase (Takara; Shiga, Japan), $2.5 \mu \mathrm{L} 10 \mathrm{X}$ PCR buffer ( $\mathrm{Mg}^{2+}$ Plus), and $18.375 \mu \mathrm{L} \mathrm{ddH_{2 }} \mathrm{O}$. The PCR procedure was $94^{\circ} \mathrm{C}$ for $5 \mathrm{~min}, 94^{\circ} \mathrm{C}$ denaturation for $30 \mathrm{~s}, 55^{\circ} \mathrm{C}$ refolding for $30 \mathrm{~s}, 72^{\circ} \mathrm{C}$ extension for $1 \mathrm{~min}$ for 35 cycles, then a $72^{\circ} \mathrm{C}$ extension for $10 \mathrm{~min}$, and storage at $4^{\circ} \mathrm{C}$. The PCR products were digested with $\mathrm{HinI}$ at $37^{\circ} \mathrm{C}$ in a water bath overnight. Digestion products were separated by $2 \%$ agarose gel electrophoresis at $150 \mathrm{~V}$ for $45 \mathrm{~min}$, and a gel imaging system (Shanghai Tianneng Technology Co.; Ltd., Shanghai, China) was used for observation.

\section{Statistical analysis}

We utilized the SPSS 17.0 software (SPSS, Inc.; Chicago, IL, USA) to analyze the data. Hardy-Weinberg equilibrium was analyzed using the $\chi^{2}$ test. Continuous data were com- 
pared using the Student $t$-test, and categorical data were compared using the $\chi^{2}$ test. Genotype and allele frequencies were compared using the $\chi^{2}$ test. Non-conditional logistic regression was used to adjust for traditional risk factors of stroke such as gender, age, body mass index, blood pressure, blood lipids, blood glucose, smoking history, history of alcohol, and other confounding factors. The odds ratio (OR) and $95 \%$ confidence interval (CI) were calculated before adjustment and after adjustment. $\mathrm{P}<0.05$ was considered to be significant.

\section{RESULTS}

\section{Hardy-Weinberg equilibrium}

The genotype and allele distributions agreed with Hardy-Weinberg equilibrium in both the ischemic patient group $\left(\chi^{2}=2.544, \mathrm{P}=0.135\right)$ and the control group $\left(\chi^{2}=1.492, \mathrm{P}=\right.$ $0.257)$. The genotying result is shown in Figure 1.

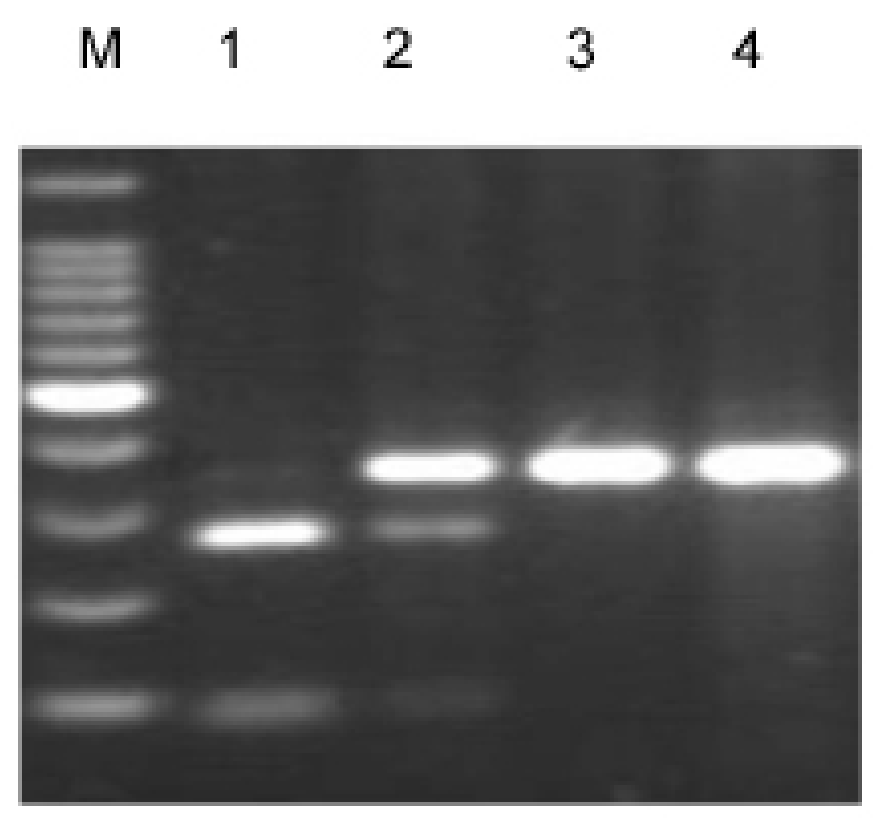

Figure 1. Electrophoresis of rs 12342 after restriction endonuclease HinI digestion. Lane $M=$ DNA marker; lane 1 $=\mathrm{GG}$ genotype; lane $2=\mathrm{AG}$ genotype; lanes 3 and $4=\mathrm{AA}$ genotype .

\section{Clinical characteristics}

Age and gender were matched in this case-control study. The body mass index showed no significant difference between the 2 groups $(\mathrm{P}>0.05)$. However, tradition risk factors of ischemic stroke such as the incidence of smoking, drinking, hypertension, diabetes, and hyperlipidemia in the ischemic stroke group was significantly higher than in the control group (all P $<0.05)$ (Table 1). 


Table 1. Characteristics of participants.
\begin{tabular}{lcrr}
\hline Characteristics & Ischemic stroke group (N =300) & Control group $(\mathrm{N}=320)$ & P value \\
\hline Age (means \pm SD, years) & $65.1 \pm 11.3$ & $65.5 \pm 11.1$ & 0.772 \\
Gender (M/F, N) & $191 / 109$ & $201 / 119$ & 0.543 \\
Body mass index (means $\left.\pm \mathrm{SD}, \mathrm{kg} / \mathrm{m}^{2}\right)$ & $24.11 \pm 4.17$ & $24.28 \pm 4.22$ & 0.132 \\
Smoking (N, \%) & $101(33.67 \%)$ & $55(18.33 \%)$ & $<0.001$ \\
Alcohol drinking (N, \%) & $108(36.00 \%)$ & $65(21.67 \%)$ & $<0.001$ \\
Hypertension (N, \%) & $209(69.67 \%)$ & $143(47.67 \%)$ & $<0.001$ \\
Diabetes (N, \%) & $164(54.67 \%)$ & $73(24.33 \%)$ & $<0.001$ \\
Hyperlipidemia (N, \%) & $132(44.00 \%)$ & $88(29.33 \%)$ & $<0.001$ \\
\hline
\end{tabular}

\section{Genotype and allele frequency distribution}

The genotype and allele frequency distribution in rs 12342 was significantly different $(\mathrm{P}<0.05)$ between the 2 groups. The AA genotype and A allele frequencies of rs 12342 in the patient group were significantly higher than those in the control group $(\mathrm{P}<0.01)$ (Table 2).

Table 2. Distribution of genotypes and alleles.
\begin{tabular}{llccc}
\hline Genotype and allele & Ischemic stroke group & Control group & P \\
\hline Genotype & AA & $118(39.3 \%)$ & $87(29.0 \%)$ & $<0.01$ \\
& AG & $128(42.7 \%)$ & $155(51.7 \%)$ & \\
Allele & GG & $54(18.0 \%)$ & $58(19.3 \%)$ & $<0.01$ \\
& A & 0.607 & 0.548 & \\
\hline
\end{tabular}

\section{Risk analysis of rs12342 and cerebral infarction}

The ischemic stroke risk of AA genotype carriers was 1.786-fold higher than that of $\mathrm{GG}$ genotype carriers $(\mathrm{OR}=1.786,95 \% \mathrm{CI}=1.432-2.775 ; \mathrm{P}=0.013)$. After adjusting for gender, age, body mass index, smoking history, alcohol consumption, hypertension, diabetes, and hyperlipidemia, as well as other confounding factors, the difference remained significant (OR $=1.874,95 \% \mathrm{CI}=1.221-2.765, \mathrm{P}=0.014)($ Table 3$)$.

Table 3. Relationship between ADIPOR2 polymorphism and ischemic stroke risk.

\begin{tabular}{lcccc}
\hline & Before-adjustment OR $(95 \% \mathrm{CI})$ & P value & After-adjustment OR $(95 \% \mathrm{CI})$ & P value \\
\hline GG & 1.00 & & & \\
GA & $1.323(0.776-2.855)$ & 0.343 & $1.432(0.798-1.988)$ & 0.765 \\
AA & $1.786(1.432-2.775)$ & 0.013 & $1.874(1.221-2.765)$ & 0.012 \\
\hline
\end{tabular}

\section{DISCUSSION}

Adiponectin is a recently discovered fat factor secreted by fat cells. It has anti-atherosclerotic and anti-inflammatory effects, increases insulin sensitivity, and maintains energy balance and glucose and lipid metabolism in addition to other important biological effects (Baranowska et al., 2011; Hewitt et al., 2012). A previous study showed that patients 
with ischemic stroke have decreased plasma adiponectin levels (Baranowska et al., 2011). In 2003, Yamauchi et al. (2003) successfully cloned adiponectin receptor cDNA for the first time, and the encoded proteins were named ADIPOR1 and ADIPOR2. Adiponectin receptors are key proteins that mediate the various biological effects of adiponectin. They are widely expressed in various tissues and organs in the human body. ADIPOR1 is mainly expressed in the skeletal muscle, while ADIPOR2 is mainly expressed in the liver (Carson et al., 2013). ADIPOR1 and ADIPOR2 are also abundantly expressed in the hypothalamus and cerebral vascular endothelial cells (Carson et al., 2013). Studies showed that adiponectin receptors are closely associated with diabetes (Pang et al., 2013), metabolic syndrome (Peters et al., 2013), and cardiovascular disease (Ding et al., 2012). They can affect cell metabolism and function and are one of the important mechanisms in the promotion and development of disease (Richards et al., 2012).

The ADIPOR 2 gene is located on chromosome 12p13.33, which includes 8 exons and 7 introns, and encodes ADIPOR2 protein containing 387 amino acids (Bermúdez et al., 2013). The rs 12342 locus is located in the 3'-untranslated region of the ADIPOR2 gene. In this study, we found that AA genotype and A allele carriers had an increased risk of ischemic stroke in a Chinese population. After infarction-type grouping, we found that the rs 12342 locus was associated with the pathogenesis of atherosclerotic cerebral infarction but not with lacunar infarction. This may be because adiponectin was closely related to atherosclerosis, which is the pathogenesis of cerebral infarction, but lacunar infarction was mainly associated with hypertension. In a recent genome-wide association study, 1 locus in chromosome 12p13, where the ADIPOR2 gene is located, was found to increase the risk of atherosclerotic cerebral infarction (Song et al., 2013). Several previous studies also found that the adiponectin gene was associated with the risk of ischemic stroke and carotid artery plaque formation in Chinese, American, Japanese, and Korean populations (Magno et al., 2008; Cheong et al., 2011; Katakami et al., 2012; Zhang et al., 2012). In other studies, the A allele and AA genotype in rs 12342 were found to increase type 2 diabetes risk in Chinese and Amish populations (Richardson et al., 2006). In addition, Richardson et al. (2006) found that triglyceride levels in AA genotype carriers of rs 12342 were significantly higher than those in GG genotype carriers, indicating that this mutation may affect lipid metabolism.

The mechanism of the association between the rs12342 polymorphism and the incidence of ischemic stroke is unclear. The rs 12342 polymorphism was found to be located in the 3 ' untranslated region of the ADIPOR2 gene. This 3' untranslated region single-nucleotide polymorphism cannot cause amino acid sequence changes. However, in many aspects, it plays an important role in regulating mRNA transcriptional processing such as post-translational modifications, intracellular localization, and transport to maintain and ensure the stability of mRNA translation efficiency, affecting the expression of 1 or more genes, and causing diseases. This locus may affect ADIPOR2 receptor expression and function through the above mechanism and affect the ability of adiponectin to perform its biological function. Furthermore, this locus may be associated with other single-nucleotide polymorphisms in linkage disequilibrium and cause changes in receptor activity. Our results must be confirmed using a larger sample size. The molecular mechanism of mutations causing the increased risk of ischemic stroke requires further analysis to determine whether mutations can lead to changes in gene expression. This will further strengthen the awareness of the pathogenesis of ischemic stroke and may lead to better disease prevention. 


\section{REFERENCES}

Baranowska B, Kochanowski J, Grudniak M, Baranowska-Bik A, et al. (2011). Decreased total serum adiponectin and its isoforms in women with acute ischemic stroke. Neuro Endocrinol. Lett. 32: 711-715.

Bermúdez VJ, Rojas E, Toledo A, Rodríguez-Molina D, et al. (2013). Single-nucleotide polymorphisms in adiponectin, AdipoR1, and AdipoR2 genes: insulin resistance and type 2 diabetes mellitus candidate genes. Am. J. Ther. 20: 414-421.

Carson BP, Del Bas JM, Moreno-Navarrete JM, Fernandez-Real JM, et al. (2013). The rab11 effector protein FIP1 regulates adiponectin trafficking and secretion. PLoS One 8: e74687.

Cheong MY, Bang OS, Cha MH, Park YK, et al. (2011). Association of the adiponectin gene variations with risk of ischemic stroke in a Korean population. Yonsei Med. J. 52: 20-25.

Ding M, Rzucidlo EM, Davey JC, Xie Y, et al. (2012). Adiponectin in the heart and vascular system. Vitam. Horm. 90: 289-319.

Du X, He S, Jiang Y, Wei L, et al. (2013). Adiponectin prevents islet ischemia-reperfusion injury through the COX2TNFalpha-NF-kappaB-dependent signal transduction pathway in mice. J. Endocrinol 218: 75-84.

Hewitt J, Castilla Guerra L, Fernández-Moreno M del C and Sierra C (2012). Diabetes and stroke prevention: a review. Stroke Res. Treat. 2012: 673187.

Hotta K, Funahashi T, Bodkin NL, Ortmeyer HK, et al. (2001). Circulating concentrations of the adipocyte protein adiponectin are decreased in parallel with reduced insulin sensitivity during the progression to type 2 diabetes in rhesus monkeys. Diabetes 50: 1126-1133.

Kabara E, Sordillo LM, Holcombe S and Contreras GA (2014). Adiponectin links adipose tissue function and monocyte inflammatory responses during bovine metabolic stress. Comp. Immunol. Microbiol. Infect. Dis. 37: 49-58.

Katakami N, Kaneto H, Matsuoka TA, Takahara M, et al. (2012). Adiponectin G276T gene polymorphism is associated with cardiovascular disease in Japanese patients with type 2 diabetes. Atherosclerosis 220: 437-442.

Kubota N, Terauchi Y, Kubota T, Kumagai H, et al. (2006). Pioglitazone ameliorates insulin resistance and diabetes by both adiponectin-dependent and -independent pathways. J. Biol. Chem. 281: 8748-8755.

Magno CP, Araneta MR, Macera CA and Anderson GW (2008). Cardiovascular disease prevalence, associated risk factors, and plasma adiponectin levels among Filipino American women. Ethn. Dis. 18: 458-463.

Pang TT, Chimen M, Goble E, Dixon N, et al. (2013). Inhibition of islet immunoreactivity by adiponectin is attenuated in human type 1 diabetes. J. Clin. Endocrinol. Metab. 98: E418-E428.

Peters KE, Beilby J, Cadby G, Warrington NM, et al. (2013). A comprehensive investigation of variants in genes encoding adiponectin (ADIPOQ) and its receptors (ADIPOR1/R2), and their association with serum adiponectin, type 2 diabetes, insulin resistance and the metabolic syndrome. BMC Med. Genet. 14: 15.

Richards JS, Liu Z, Kawai T, Tabata K, et al. (2012). Adiponectin and its receptors modulate granulosa cell and cumulus cell functions, fertility, and early embryo development in the mouse and human. Fertil. Steril. 98: 471-479.

Richardson DK, Schneider J, Fourcaudot MJ, Rodriguez LM, et al. (2006). Association between variants in the genes for adiponectin and its receptors with insulin resistance syndrome (IRS)-related phenotypes in Mexican Americans. Diabetologia 49: 2317-2328.

Song W, Huo T, Guo F, Wang H, et al. (2013). Globular adiponectin elicits neuroprotection by inhibiting NADPH oxidasemediated oxidative damage in ischemic stroke. Neuroscience 248C: 136-144.

Tian L, Luo N, Zhu X, Chung BH, et al. (2012). Adiponectin-AdipoR1/2-APPL1 signaling axis suppresses human foam cell formation: differential ability of AdipoR1 and AdipoR2 to regulate inflammatory cytokine responses. Atherosclerosis 221: 66-75.

Uemura Y, Shibata R, Ohashi K, Enomoto T, et al. (2013). Adipose-derived factor CTRP9 attenuates vascular smooth muscle cell proliferation and neointimal formation. FASEB J. 27: 25-33.

Wahab F, Bano R, Jabeen S, Irfan S, et al. (2010). Effect of peripheral kisspeptin administration on adiponectin, leptin, and resistin secretion under fed and fasting conditions in the adult male rhesus monkey (Macaca mulatta). Horm. Metab. Res. 42: 570-574.

Yamauchi T, Kamon J, Ito Y, Tsuchida A, et al. (2003). Cloning of adiponectin receptors that mediate antidiabetic metabolic effects. Nature 423: 762-769.

Yang J, Lin SC, Chen G, He L, et al. (2013). Adiponectin promotes monocyte-to-fibroblast transition in renal fibrosis. $J$. Am. Soc. Nephrol. 24: 1644-1659.

Zhang BC, Liu WJ, Che WL and Xu YW (2012). Serum total adiponectin level and risk of cardiovascular disease in Han Chinese populations: a meta-analysis of 17 case-control studies. Clin. Endocrinol. 77: 370-378. 